\title{
Transcriptome profiling reveals the roles of pigment mechanisms in postharvest broccoli yellowing
}

\author{
Feng Luo ${ }^{1}$, Jia-Hui Cai ${ }^{1}$, Xi-Man Kong ${ }^{1}$, Qian Zhou ${ }^{1}$, Xin Zhou' ${ }^{1}$ Ying-Bo Zhao ${ }^{1}$ and Shu-Juan Ji ${ }^{1}$
}

\begin{abstract}
Postharvest broccoli is prone to yellowing during storage, which is the key factor leading to a reduction in value. To explore appropriate control methods, it is important to understand the mechanisms of yellowing. We analyzed the genes related to the metabolism of chlorophyll, carotenoids, and flavonoids and the transcription factors (TFs) involved in broccoli yellowing using transcriptome sequencing profiling. Broccoli stored at $10^{\circ} \mathrm{C}$ showed slight yellowing on postharvest day 5 and serious symptoms on day 12. There were significant changes in chlorophyll fluorescence kinetics, mainly manifesting as a decrease in the Fv/Fm value and an increase in nonphotochemical quenching, during the yellowing process. Transcriptome sequencing profiles from samples of fresh broccoli and broccoli with slight and severe yellowing revealed 6, 5, and 4 differentially expressed genes involved in chlorophyll metabolism, carotenoid biosynthesis, and flavonoid biosynthesis, respectively. The transcription factor gene ontology categories showed that the MYB, bHLH, and bZip gene families were involved in chlorophyll metabolism. In addition, the transcription factor families included NACs and ethylene response factors (ERFs) that regulated carotenoid biosynthesis. Reverse transcription polymerase chain reaction further confirmed that bHLH66, PIF4, LOB13, NAC92, and APL were vital transcription factors that potentially regulated the CAO and HYD genes and were involved in chlorophyll metabolism and the carotenoid biosynthetic process. The flavonoid biosynthetic pathway was mainly regulated by MYBs, NACs, WRKYs, MADSs, and bZips. The results of the differentially expressed gene (DEG) and pigment content analyses indicated that the transcriptome data were accurately and positively associated with broccoli yellowing.
\end{abstract}

\section{Introduction}

The edible part of broccoli is a green cluster of flowers that is composed of dense buds and stems. Broccoli (Brassica oleracea L. var. italica) is a vegetable with high nutritional value and notable anticancer properties ${ }^{1}$. However, the inflorescences and stems of postharvest broccoli are prone to wilting and yellowing, which greatly influences product quality and value. Therefore, understanding the yellowing mechanisms and their regulation is of considerable theoretical and practical importance.

\footnotetext{
Correspondence: Shu-Juan Ji (jsjsyau@yeah.net) (jsjsyau@sina.com) ${ }^{1}$ Department of Food Science, Shenyang Agricultural University, 110866 Shenyang, PR China
}

Various factors can induce postharvest yellowing of fruits and vegetables, including senescence, pathogen infection (yellow leaf curl virus and chlorosis virus), free radicals, energy, and metal ions. Maldonado-Torres et al. ${ }^{2}$ found that $\mathrm{Fe}^{2+}$ and $\mathrm{Mn}^{2+}$ induced yellowing of Mexican lime leaves and simultaneously changed the anatomy of the leaves. Hydrangea chlorotic mottle virus (HdCMV) can lead directly to yellowing of hydrangeas. In addition, some secondary metabolites in fruits and vegetables affect the sensory color of plants. Cadrain ${ }^{3}$ found that the yellowing of Arabidopsis thaliana leaves is caused by chlorophyll degradation and anthocyanin accumulation. Thomas ${ }^{4}$ found that chlorophyll degradation was the major factor in the yellowing of a Festuca pratensis Huds.

\section{(c) The Author(s) 2019}

(c) (i) Open Access This article is licensed under a Creative Commons Attribution 4.0 International License, which permits use, sharing, adaptation, distribution and reproduction c. in any medium or format, as long as you give appropriate credit to the original author(s) and the source, provide a link to the Creative Commons license, and indicate if changes were made. The images or other third party material in this article are included in the article's Creative Commons license, unless indicated otherwise in a credit line to the material. If material is not included in the article's Creative Commons license and your intended use is not permitted by statutory regulation or exceeds the permitted use, you will need to obtain permission directly from the copyright holder. To view a copy of this license, visit http://creativecommons.org/licenses/by/4.0/. 
0 d

a

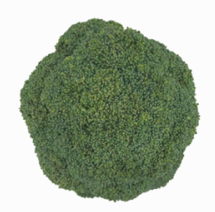

$5 \mathbf{d}$

b

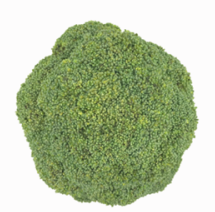

$12 \mathrm{~d}$

c
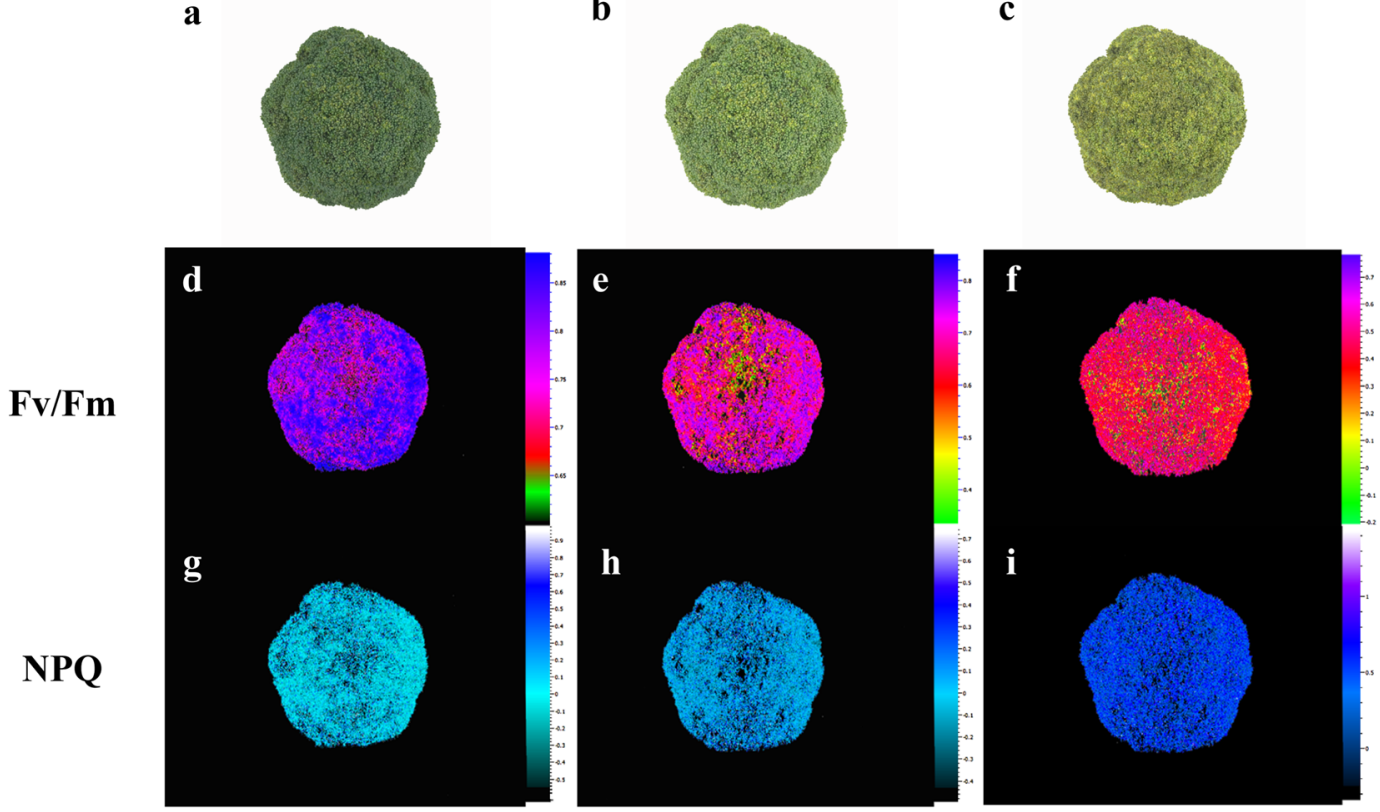

Fig. 1 Chlorophyll fluorescence imaging of broccoli. $\mathbf{a}-\mathbf{c}$ The sensory images obtained during broccoli yellowing. $\mathbf{d}-\mathbf{i}$ Fluorescence images corresponding to the Fv/Fm $(\mathbf{d}-\mathbf{f})$ and NPQ $(\mathbf{g}-\mathbf{i})$ values. The images in $(\mathbf{d}-\mathbf{i})$ were normalized to a false color bar. Determination of the Fv/Fm ratio was carried out on dark-adapted broccoli for 15 min, while NPQ analysis was conducted at the optimum light saturation intensity

mutant. In pepper $^{5}$ (Capsicum sp.), there is a correlation between capsanthin-capsorubin synthase and fruit color. Several enzymes are involved in catalyzing pigment metabolism; however, changes in plant color are usually caused by specific genes ${ }^{6}$. Previous studies ${ }^{7}$ showed that ethylene treatment significantly contributes to chlorophyll a degradation and yellowing, while 1-MCP treatment significantly delays the onset of yellowing. Wang et al. ${ }^{8}$ revealed that a modified atmosphere could inhibit the activity of respiratory enzymes and delay the yellowing of harvested broccoli. Moreover, electrostatic atomized water particle treatment ${ }^{9}$ and N6-benzylaminopurine treatment $^{10}$ could inhibit chlorophyll degradation and carotenoid biosynthesis.

High-throughput RNA-seq is an effective and sensitive technology that can reveal comprehensive genic changes in plants. In recent years, transcriptome sequencing has significantly advanced molecular-level research on higher plants. In pomelo ${ }^{11}$ (Citrus grandis), cherry ${ }^{12}$ (Prunus avium L.), apple ${ }^{13}$, wheat ${ }^{14}$ (Triticum aestivum L.), and Brassica napus ${ }^{15}$, previous studies using transcriptome analysis have reported some key genes and transcriptome factors (TFs) involved in color change events. However, there are few reports on transcriptome analysis of broccoli, except for a study on cytokinin-treated broccoli based on proteomics and transcriptomics ${ }^{16}$.
In this study, high-throughput sequencing was employed to characterize the mRNA information of fresh, slightly yellowed, and severely yellowed postharvest broccoli. We conducted a pigment and chlorophyll fluorescence study of the yellowing process, and the chlorophyll metabolism and carotenoid and flavonoid biosynthetic pathways associated with broccoli yellowing were identified. Subsequently, we determined the major genes for key pathways. The upstream TFs that regulated the yellowing-related metabolic pathways were identified by analyzing the gene ontology (GO) categories. Our research aimed to explore the vital metabolic pathways in the process of broccoli yellowing and identify the key genes and their potential upstream regulatory factors.

\section{Results}

Changes in chlorophyll fluorescence kinetic parameters and images during broccoli yellowing

$\mathrm{Fv} / \mathrm{Fm}$ is a measurement ratio that represents the maximum potential efficiency of photosystem II if all capable reaction centers are open, and nonphotochemical quenching (NPQ) is used to quantify photoprotective and photoinhibitory processes. By measuring chlorophyll fluorescence during broccoli yellowing, we obtained kinetic parameters (Table S1) and images (Fig. 1). The results showed that broccoli heads stored at $10{ }^{\circ} \mathrm{C}$ began 
to yellow on day 5 and showed severe yellowing on day 12 (Fig. 1a-c). During broccoli yellowing, the Fv/Fm values decreased sharply, while the NPQ values increased.

\section{Transcriptomic changes in broccoli during yellowing}

Based on the chlorophyll fluorescence kinetic parameters, transcriptome sequencing results were obtained from three broccoli samples (fresh, slightly yellowed, and severely yellowed) with three biological replicates for each sample. A total of $68.06 \mathrm{~Gb}$ of clean data were obtained, and the Q30 base percentage was greater than $94.73 \%$. Clean reads were sequentially compared with the Brassica oleracea genomic database, with match ratios ranging from 83.11 to $85.93 \%$. Based on the comparison results, gene expression was analyzed using fragments per kilobase of transcript per million mapped reads (FPKM), and differentially expressed genes (DEGs) were identified according to their expression levels in different samples. As shown in Table S2, there were more DEGs in the $0 \mathrm{~d}$ vs. $5 \mathrm{~d}$ set than in the $0 \mathrm{~d}$ vs. $12 \mathrm{~d}$ set, and more DEGs appeared at $0 \mathrm{~d}$ than at $5 \mathrm{~d}$ or $12 \mathrm{~d}$. The DEGs were matched and assigned to 92 KEGG pathways.

Studies on color have revealed that, in addition to DEGs, TFs play key roles in modulating color changes and the activity of pigment biosynthetic pathways. In our analysis of the transcriptome data, we focused on differentially expressed TFs. The MYB and NAC families were the most abundant TF families during the broccoli yellowing process, followed by the AP2/ERF, bHLH, WRKY, B3, and LOB families (Table 1); many of these were expressed before the samples began to yellow. Additionally, bHLHs and WRKYs were mostly upregulated before yellowing, while almost all of the abovementioned families were downregulated in the later stages of yellowing. In the preyellowing stage, $89 \%$ of B3s were downregulated, and only 2 upregulated B3s were detected when the samples yellowed. LOBs were upregulated and only detected in samples that had not yellowed. The number of AP2/ERFs was reduced by $68 \%$ after day 5 .

\section{Chlorophyll metabolism genes involved in broccoli coloration during yellowing}

Six DEGs, which are the key candidate genes encoding enzymes, were associated with chlorophyll metabolism (Fig. 2a). In the process of broccoli yellowing, the expression of CHLI (Bo7g109930 and Bo1g039660) and POR (Bo8g002560 and Bo5g003990) genes was continuously downregulated, while that of two $C A O$ genes (Bo6g003400 and Bo8g021880) was significantly upregulated. We also found that the expression of two NYC1 (Bo4g133190 and Bo8g033750) genes and the HO1 (Bo5g057380) gene was significantly upregulated in early yellowing but slightly downregulated in the later stages of yellowing. The change in the expression of the $C P O X$
(Bo8g116050) gene showed exactly the opposite pattern to that of NYC1 and HO1.

\section{Carotenoid biosynthesis genes involved in broccoli coloration during yellowing}

As shown in Fig. 2b, five DEGs were annotated as key genes encoding enzymes related to carotenoid biosynthesis. The DEGs encoding HYD (Bo7g110490) were differentially expressed in multiple branches of this KEGG pathway and were upregulated during broccoli yellowing. Additionally, the DEGs encoding ZEP were significantly upregulated before broccoli yellowed but were then downregulated. During the process of broccoli yellowing, LCYE (Bo3g019500 and Bo5g103680), VDE (Bo5g009080), and CCS (Bo6g122000) were significantly downregulated, but CCS (Bo1g019460) was not.

\section{Flavonoid biosynthesis genes involved in broccoli coloration during yellowing}

As shown in Fig. 2c, four DEGs were annotated in the flavonoid biosynthesis pathway; however, most secondary metabolic pathways were weakened by the downregulation of genes, including CHS (Bo3g009440 and Bo9g166290), FLS (Bo8g076760), and F3H (Bo8g081770), during broccoli yellowing. We found that $4 C L$ (Bo4g173070) was the only gene that was continuously upregulated during the yellowing process. However, another 4CL gene (Bo3g024650) was significantly downregulated during the early stage of yellowing but was upregulated after day 5 .

\section{TFs involved in broccoli coloration}

As shown in Table 2, the TF families involved in chlorophyll metabolism, carotenoid biosynthesis, and flavonoid biosynthesis were relatively fixed and were involved in the biological process regardless of the stage of broccoli yellowing. MYBs, bHLHs, and bZips were the candidate TFs involved in regulating the chlorophyll metabolism-related pathway. NACs, bHLHs, bZips, MYBs, and ERFs have the potential to participate in the regulation of the carotenoid biosynthetic pathway. Moreover, six potential TF families involved in the regulation of flavonoid biosynthetic pathways were identified, including the MYB, NAC, WRKY, MADS, and bZip families.

\section{Pigment changes in broccoli}

The yellowing process of broccoli was accompanied by the evolution of pigment content. As shown in Table 3, the chlorophyll $a$ content gradually decreased, and the chlorophyll $b$ content fluctuated. However, the contents of the four carotenoid components gradually increased, especially after day 5 . The contents of zeaxanthin, $\beta$-carotene, lutein, and $\beta$-cryptoxanthin increased by 
Table 1 Differentially expressed transcription factors (TFs) in broccoli

\begin{tabular}{|c|c|c|c|c|c|}
\hline DEG set & TF family & Number of DEGs & Upregulated & Downregulated & Description \\
\hline \multirow[t]{16}{*}{$0 d$ vs. $5 \mathrm{~d}$} & AP2/ERF & 50 & 14 & 36 & Ethylene-responsive transcription factor \\
\hline & B3 & 27 & 3 & 24 & B3 DNA-binding domain \\
\hline & $\mathrm{bHLH}$ & 46 & 16 & 30 & Helix-loop-helix DNA-binding domain \\
\hline & bZip & 17 & 9 & 8 & Basic region leucine zipper \\
\hline & $\mathrm{C} 2 \mathrm{C} 2$ & 34 & 11 & 23 & Zinc finger protein \\
\hline & $\mathrm{C} 2 \mathrm{H} 2$ & 12 & 6 & 6 & Zinc finger $\mathrm{C} 2 \mathrm{H} 2$ domain-containing protein \\
\hline & $\mathrm{C} 3 \mathrm{H}$ & 7 & 3 & 4 & Zinc finger $\mathrm{CCCH}$ domain-containing protein \\
\hline & $\mathrm{HB}-\mathrm{HD}$ & 25 & 9 & 16 & Homeobox domain \\
\hline & HSF & 5 & 5 & 0 & Heat stress transcription factor \\
\hline & LOB & 12 & 11 & 1 & LOB domain-containing protein \\
\hline & MADS & 11 & 6 & 5 & SRF-type transcription factor \\
\hline & MYB & 68 & 41 & 27 & MYB-related protein \\
\hline & NAC & 57 & 47 & 10 & NAC domain-containing protein \\
\hline & WRKY & 42 & 31 & 11 & WRKY DNA -binding domain \\
\hline & other TFs & 48 & 30 & 18 & \\
\hline & total & 461 & 242 & 219 & \\
\hline \multirow[t]{16}{*}{$5 \mathrm{~d}$ vs. $12 \mathrm{~d}$} & AP2/ERF & 16 & 6 & 10 & Ethylene-responsive transcription factor \\
\hline & B3 & 2 & 2 & 0 & B3 DNA-binding domain \\
\hline & $\mathrm{bHLH}$ & 14 & 6 & 8 & Helix-loop-helix DNA-binding domain \\
\hline & bZip & 4 & 3 & 1 & Basic region leucine zipper \\
\hline & $\mathrm{C} 2 \mathrm{C} 2$ & 8 & 8 & 0 & Zinc finger protein \\
\hline & $\mathrm{C} 2 \mathrm{H} 2$ & 1 & 0 & 1 & Zinc finger $\mathrm{C} 2 \mathrm{H} 2$ domain-containing protein \\
\hline & $\mathrm{C} 3 \mathrm{H}$ & 1 & 1 & 0 & Zinc finger $\mathrm{CCCH}$ domain-containing protein \\
\hline & $\mathrm{HB}-\mathrm{HD}$ & 3 & 2 & 1 & Homeobox domain \\
\hline & HSF & 3 & 2 & 1 & Heat stress transcription factor \\
\hline & LOB & 0 & 0 & 0 & LOB domain-containing protein \\
\hline & MADS & 1 & 1 & 0 & SRF-type transcription factor \\
\hline & MYB & 10 & 6 & 4 & MYB-related protein \\
\hline & NAC & 4 & 1 & 3 & NAC domain-containing protein \\
\hline & WRKY & 21 & 5 & 16 & WRKY DNA -binding domain \\
\hline & other TFs & 29 & 8 & 21 & \\
\hline & Total & 117 & 51 & 66 & \\
\hline
\end{tabular}

FDR $<0.05$ and $\mid$ log2 (fold change) $\mid \geq 1$ were set as the threshold values for significantly different expression

$71.7 \%, 178.5 \%, 63.4 \%$, and $703.7 \%$, respectively, between 5 $\mathrm{d}$ and $12 \mathrm{~d}$. The flavonoid content changed primarily at the stage of slight yellowing, decreasing by $26.7 \%$; subsequently, there was no significant change.

Validation of DEG expression in the transcriptome data

To validate the key DEG results (Fig. 3), the 21 DEGs that showed the most significant differences in expression were selected: six chlorophyll metabolism pathway genes (Fig. 3a-f), five carotenoid biosynthetic pathway genes (Fig. $3 g-k$ ), four flavonoid biosynthetic pathway genes (Fig. 3l-o), and six TFs (Fig. 3p-u). We analyzed the evolution of expression during broccoli yellowing using RT-PCR. The mRNA-seq and RT-PCR data were very closely correlated, and there was high consistency in the up- and downregulated expression of DEGs. 
a Chlorophyll metabolism

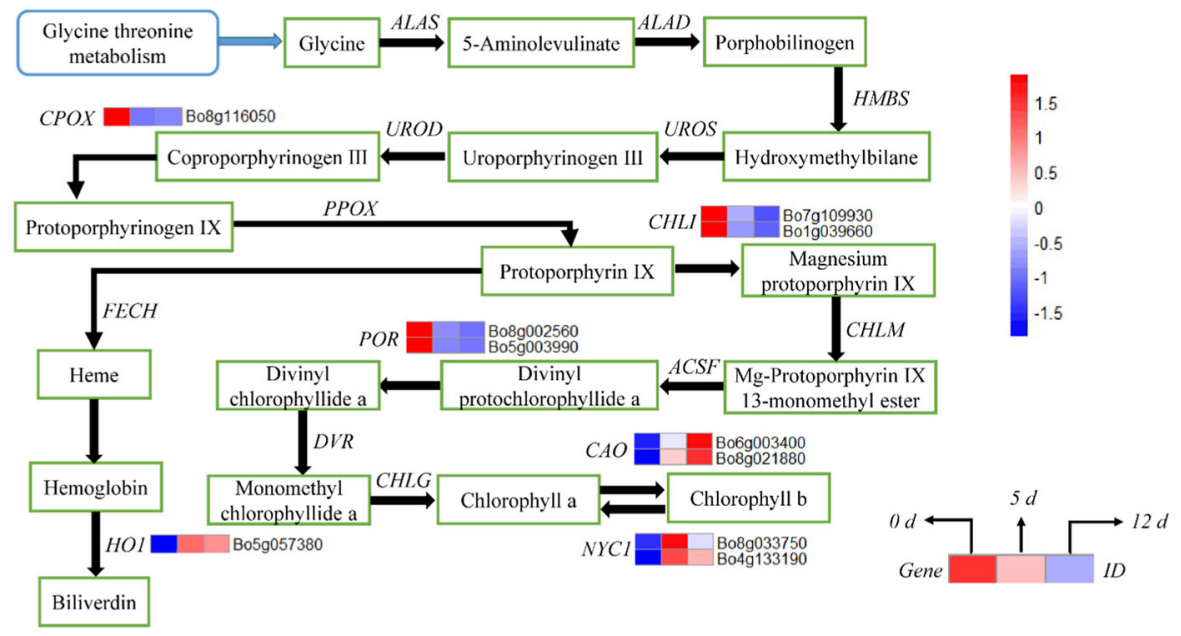

b Carotenoid biosynthesis
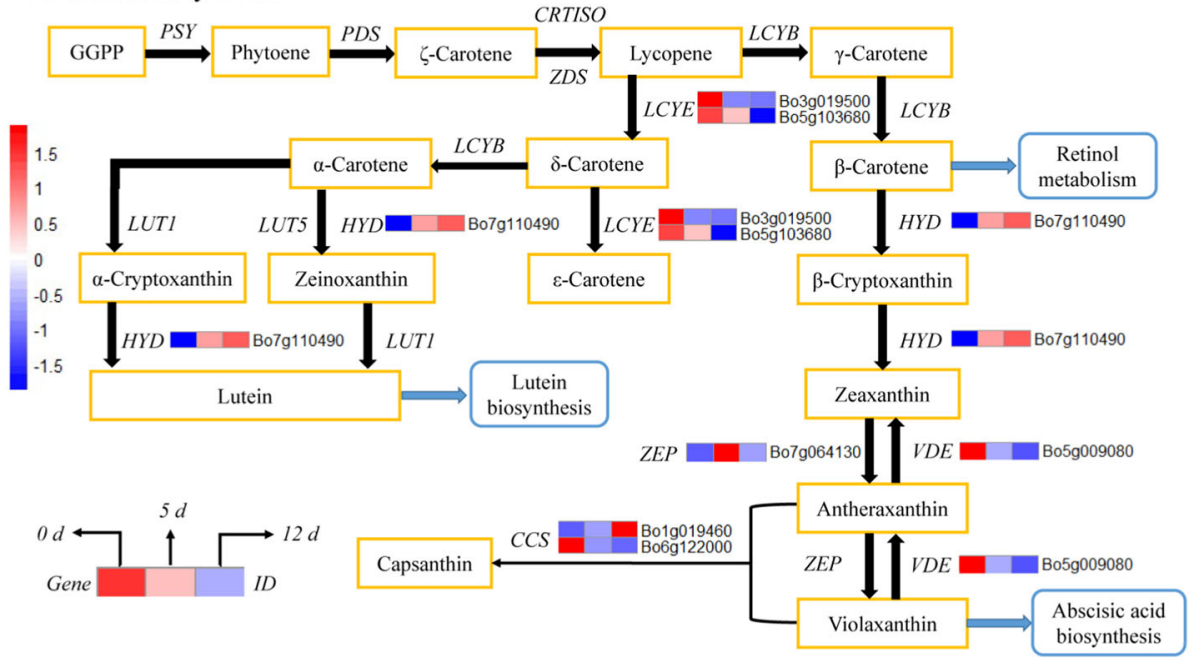

c Flavonoid biosynthesis

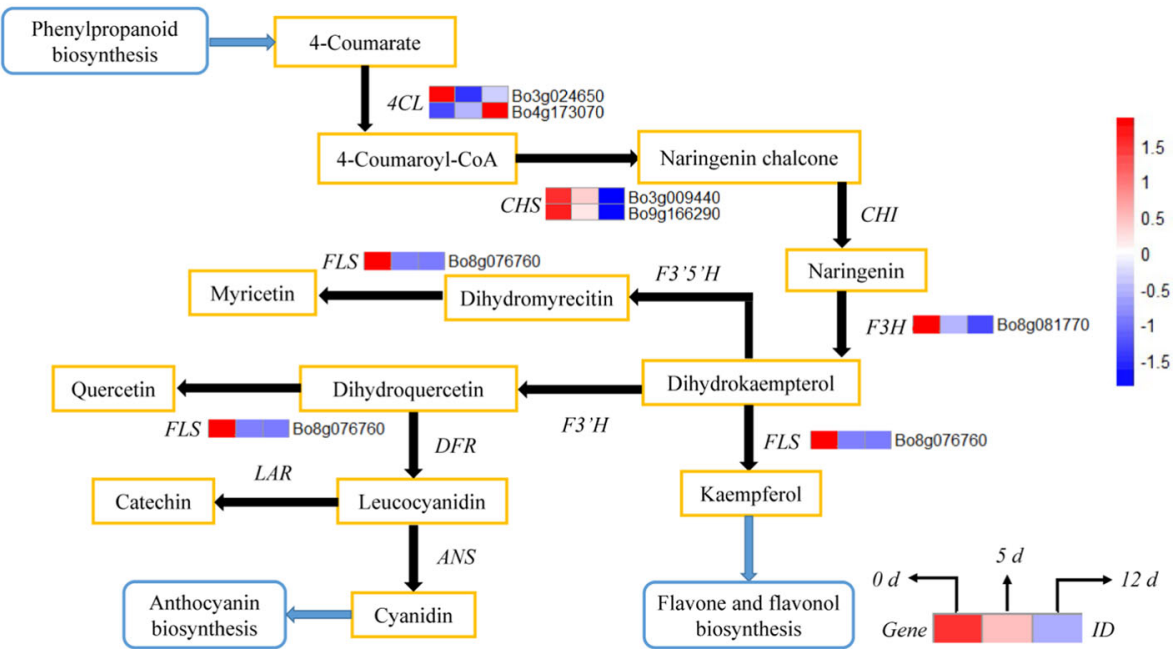

Fig. 2 (See legend on next page.) 


\begin{abstract}
(see figure on previous page)
Fig. 2 Transcriptional profiling of differentially expressed genes (DEGs) associated with pigment metabolism in broccoli during yellowing. The mean FPKM (fragments per kilobase of transcript per million mapped reads) values for the DEGs were calculated from three biological replicates for each sampling point $(0,5$, and $12 \mathrm{~d}$ post harvest). The progression of the color scale from blue to red represents an increase in the FPKM values. a The DEGs involved in chlorophyll metabolism. ALAS 5-aminolevulinate synthase, ALAD porphobilinogen synthase, HMBS hydroxymethylbilane synthase, UROS uroporphyrinogen-III synthase; UROD uroporphyrinogen decarboxylase, CHLI magnesium chelatase subunit I, HO1 heme oxygenase 1, CHLM magnesium-protoporphyrin O-methyltransferase, CAO chlorophyllide a oxygenase; HEMF coproporphyrinogen III oxidase, DVR divinyl chlorophyllide a 8-vinyl-reductase, FECH protoporphyrin/coproporphyrin ferrochelatase, PPOX protoporphyrinogen/coproporphyrinogen III oxidase, NYC1 chlorophyll(ide) $b$ reductase, ACSF magnesium-protoporphyrin IX monomethyl ester (oxidative) cyclase, POR protochlorophyllide reductase, CHLG chlorophyll/bacteriochlorophyll a synthase. b The DEGs involved in carotenoid biosynthesis. PSY phytoene synthase, ZDS $\zeta$-carotene desaturase, LCYB lycopene $\beta$-cyclase, LCYE lycopene $\varepsilon$-cyclase, HYD $\beta$-carotene 3-hydroxylase, ZEP zeaxanthin epoxidase, VDE violaxanthin de-epoxidase, LUT1 carotene epsilon-monooxygenase, LUT5 beta-ring hydroxylase, PDS 15-cis-phytoene desaturase, CRTISO prolycopene isomerase, CCS capsanthin/ capsorubin synthase. $\mathbf{c}$ The DEGs involved in flavonoid biosynthesis. 4CL 4-coumarate CoA ligase, CHS chalcone synthase, CHI chalcone isomerase, F3H flavanone 3-hydroxylase, $F^{\prime} 5^{\prime} H$ flavonoid 3',5'-hydroxylase, LAR leucocyanidin reductase, F3'H flavonoid 3'-hydroxylase, FLS flavonol synthase, DFR dihydroflavonol 4-reductase, ANS anthocyanidin synthase
\end{abstract}

\section{Discussion}

\section{Evolution of pigments during broccoli yellowing}

Many factors are involved in postharvest broccoli yellowing. In the final analysis, the evolution of the content and the proportions of pigments were concordant. Considering these results, we suggest that early yellowing can be attributed to the accumulation of chlorophyll $b$ and the lack of chlorophyll $a$, which leads to broccoli continuing to yellow after day 5 . The $\mathrm{Fv} / \mathrm{Fm}$ values decreased with broccoli yellowing, which reflected the gradual destruction of photosystem II (PSII) in the plant. The chloroplast thylakoid membrane is the main site of PSII, and its structure may be destroyed during the yellowing process, which would lead to a further decrease in the content of chlorophyll components ${ }^{17}$. Briefly, changes in chlorophyll components contributed to broccoli color variation at the pigment level.

The contents of the main carotenoid components in broccoli- $\beta$-carotenoid, lutein, and $\beta$-cryptoxanthincontinued to increase throughout the yellowing process. In particular, significant increases in the $\beta$-carotenoid and $\beta$-cryptoxanthin content were detected after day 5 . Chlorophyll deficiency and carotenoid accumulation were the main reasons for broccoli yellowing. The observed increase in NPQ during the yellowing process might be due to the limitation of the photosynthetic substrate after harvest. The light energy used by broccoli is reduced, and a large part of the absorbed light quantum is dissipated in the form of heat energy and is not used to drive photosynthesis ${ }^{18}$. The dissipation of excess energy protects the photosystem, allowing the original electron acceptors of PSII to maintain a high oxidation state, thereby preventing damage to broccoli by the optical system. The xanthophyll cycle is one of the most important ways to release excess energy, mainly through the two-stage decyclization of violaxanthin, which was another reason for the increase in zeaxanthin content ${ }^{19}$.
Flavonoids have been widely studied as important yellow pigments in many fruits and vegetables; the results of these studies revealed that flavonoids are the main contributors to the yellow color of ginkgo ${ }^{20}$ and that they play a key role in the color of Arabidopsis thalian $a^{21}$ and sweet orange during the process of fruit development and ripening. However, the decline in flavonoid levels did not contribute to the yellowing of broccoli before day 5 . Although flavonoids contain flavonols, anthocyanins, and flavones, which contribute to coloration, flavonoids did not appear to be a major factor in broccoli yellowing.

\section{DEGs involved in chlorophyll metabolism}

Chlorophyll metabolism occurs in the pigment-proteolipid complexes within thylakoid membranes that are organized to ensure a stable order of the branches in the complex chlorophyll metabolism pathway. This order is achieved by the regulation of genes involved in the pathway. Based on the transcriptome data, $C A O$ was continuously upregulated throughout the process of broccoli yellowing, resulting in continuous transformation of chlorophyll $a$ into chlorophyll $b$ via catalysis by CAO, which resulted in chlorophyll $a$ degradation. Equally significantly, chlorophyll $b$ was reconverted to chlorophyll $a$ via catalysis by NYC1. We found that NYC1 was significantly upregulated before yellowing but was downregulated after day 5 . The present study showed that the expression of $N Y C 1$ and $C A O$ exhibited a strong correlation with chlorophyll $b$ accumulation and chlorophyll a degradation during broccoli yellowing. This phenomenon was the result of the ability of CAO to catalyze chlorophyll $b$ synthesis, overcoming the degradation of chlorophyll $b$ by NYC1. Therefore, we conclude that $C A O$ plays an important role in the chlorophyll metabolism pathway during broccoli yellowing. However, this result differs from previous reports on broccoli yellowing, which concluded that $C H L I$ and $P A O$ were the key 


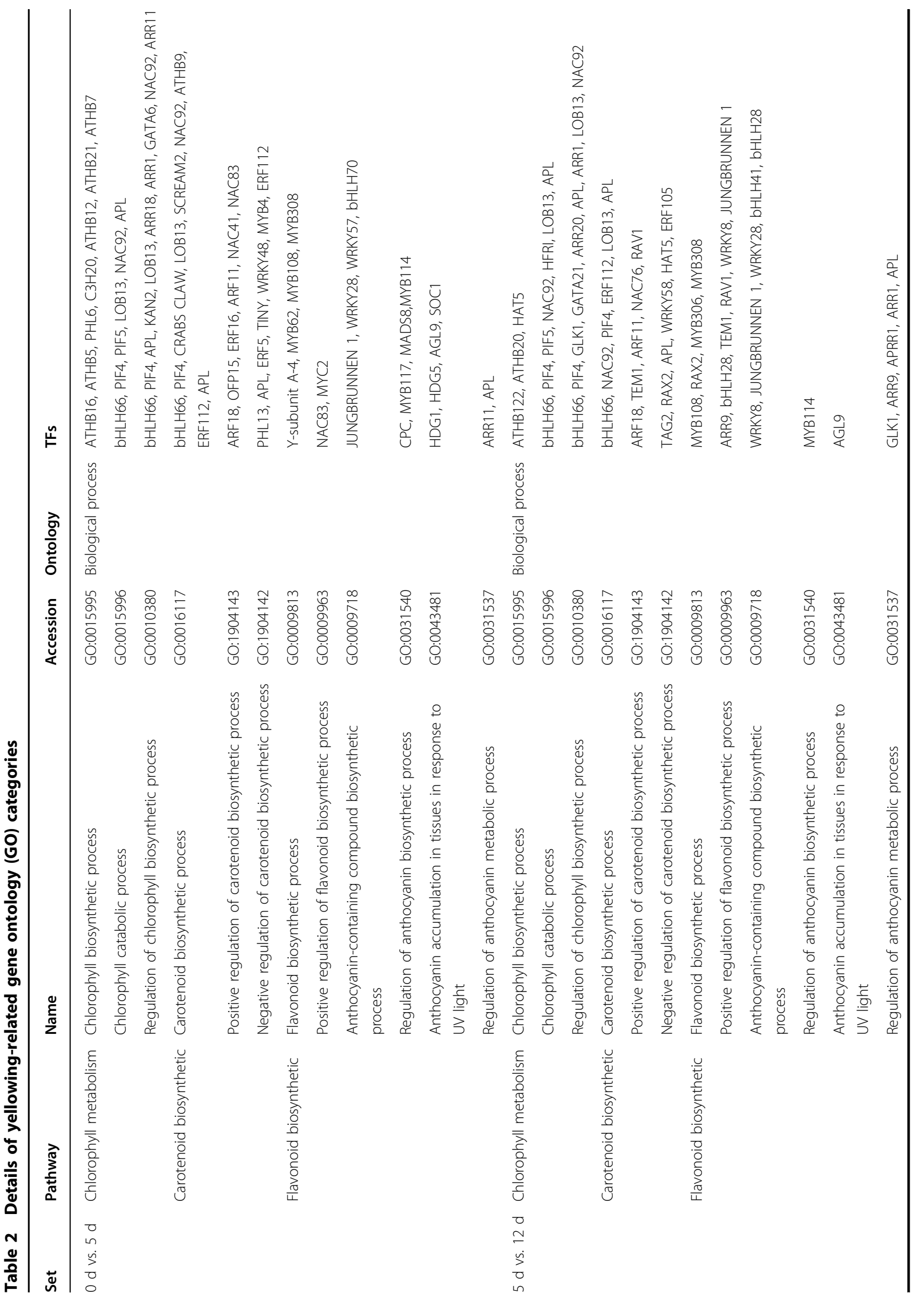


Table 3 Evolution of pigment contents in broccoli during yellowing

\begin{tabular}{llll}
\hline Pigment content & \multicolumn{3}{l}{ Storage time (d) } \\
\cline { 2 - 4 } & $\mathbf{0 ~ d}$ & $\mathbf{5 ~ d}$ & $\mathbf{1 2 ~ d}$ \\
\hline Chlorophyll content & & & \\
Chlorophyll $a(\mathrm{mg} / \mathrm{kg})$ & $1.12 \pm 0.31 \mathrm{a}$ & $0.77 \pm 0.26 \mathrm{~b}$ & $0.50 \pm 0.21 \mathrm{c}$ \\
Chlorophyll $b(\mathrm{mg} / \mathrm{kg})$ & $0.23 \pm 0.11 \mathrm{~b}$ & $0.38 \pm 0.10 \mathrm{a}$ & $0.18 \pm 0.06 \mathrm{c}$ \\
Carotenoid content & & & \\
Zeaxanthin $(\mathrm{mg} / \mathrm{kg})$ & $1.08 \pm 0.05 \mathrm{c}$ & $2.12 \pm 0.81 \mathrm{~b}$ & $3.64 \pm 1.01 \mathrm{a}$ \\
$\beta$-Carotene $(\mathrm{mg} / \mathrm{kg})$ & $3.81 \pm 1.14 \mathrm{c}$ & $7.24 \pm 2.26 \mathrm{~b}$ & $20.16 \pm 6.02 \mathrm{a}$ \\
Lutein $(\mathrm{mg} / \mathrm{kg})$ & $5.62 \pm 1.02 \mathrm{~b}$ & $6.12 \pm 2.11 \mathrm{~b}$ & $10.01 \pm 2.54 \mathrm{a}$ \\
$\beta$-Cryptoxanthin $(\mu \mathrm{g} / \mathrm{kg})$ & $14.21 \pm 2.12 \mathrm{c}$ & $26.18 \pm 5.17 \mathrm{~b}$ & $210.4 \pm 16.20 \mathrm{a}$ \\
Flavonoid content & & & \\
Flavonoids $(\mu \mathrm{g} / \mathrm{kg})$ & $15.72 \pm 2.62 \mathrm{a}$ & $12.41 \pm 2.07 \mathrm{~b}$ & $13.66 \pm 3.28 \mathrm{~b}$ \\
\hline
\end{tabular}

The values represent the means \pm standard deviations of three experiments, each with three biological replicates $(n=9)$. The different letters within the rows indicate significant differences among different storage times $(P<0.05)$

genes in the chlorophyll metabolism pathway ${ }^{22}$. Additionally, we proposed that $\mathrm{HO} 1$ contributed to the coloration of broccoli during yellowing. Heme oxygenase (HO) can catalyze a large amount of hemoglobin to biliverdin ${ }^{23}$, releasing $\mathrm{CO}$ and $\mathrm{Fe}^{2+}$, which accumulate in dark green biliverdin. $\mathrm{HO}$ was identified from the study of neonatal jaundice and contributes to the coloration of bird feathers and eggshells. Recently, researchers found that $\mathrm{HO}$ is involved in the regulation of pigment formation in plants. In Brassica napus ${ }^{24}$, defects in $\mathrm{HO} 1$ could lead to a decrease in chlorophyll content. Mahawar and Shekhawat ${ }^{25}$ found that $\mathrm{HO}$ is a functionally diverse enzyme in photosynthetic organisms that plays vital roles in defense mechanisms, phytochrome chromophore biosynthesis, and cellular signaling. $\mathrm{HO}^{26}$ is also involved in the regulation of the anti-inflammatory activity of rhein in Cassia fistula L. HO1, as a DEG, was significantly upregulated before broccoli began to yellow and was then downregulated after day 5 . This result indicates that the green pigment in broccoli is persistently synthesized during the yellowing process of broccoli and that its synthesis is gradually inhibited after yellowing begins. Collectively, these results show that chlorophyll accumulation and composition during broccoli yellowing is highly regulated by the coordinated transcriptional activation of chlorophyll metabolism genes, as has been demonstrated in some fruits.

\section{DEGs involved in carotenoid biosynthesis}

Carotenoids-a large family of pigments that range in color from yellow to red-accumulate in large amounts in ripe fruit and senescent vegetables. However, the patterns of carotenoid biosynthesis in different species are very variable. In red-flesh navel orange, grapefruit, and pomelo, lycopene accumulation is a characteristic of carotenoid biosynthesis ${ }^{27}$. In our study, lutein biosynthesis, retinol metabolism, and the abscisic acid biosynthetic pathway were mainly involved in broccoli yellowing.

In addition, the key genes that influence carotenoid biosynthesis can vary among species. The expression of the 1-deoxy-D-xylulose-5-phosphate synthase $(D X S)$ and hydroxymethylbutenyl diphosphate reductase $(H D R)$ genes is directly related to carotenoid accumulation during tomato fruit ripening ${ }^{28}$. In ginkgo biloba, the upregulated genes $Z$-ISO, ZDS, and $L C Y E$ enhance carotenoid accumulation. In the present study, six DEGs were identified downstream of lycopene biosynthesis. However, PSY is the most important rate-limiting enzyme in the carotenoid biosynthesis pathway and was not differentially expressed upstream of lycopene biosynthesis during broccoli yellowing. The explanation for this observation might be that the key areas for broccoli yellowing were within and downstream of the carotenoid biosynthetic pathway.

$\beta$-Carotenoid is the most abundant carotenoid component in broccoli, and the reason for its continuous increase might be the role of $L C Y B$ regulation, which could offset $H Y D$ upregulation as a $\beta$-carotenoid downstream gene. Compared with the accumulation of other carotenoid components, the massive accumulation of $\beta$-carotenoid during yellowing appeared to correlate well with the changes in broccoli coloration. Cyclization of lycopene is a branch point: one branch leads to $\beta$-carotene; the other, $\alpha$-carotene. Furthermore, the end of the $\alpha$-carotene metabolic pathways points to lutein biosynthesis. The transcriptome data indicated that the only DEG in the $\alpha$-carotene branch was $H Y D$, and the expression of $H Y D$ led to noticeable accumulation of lutein after day 5 . The transformation of $\beta$-carotene into $\beta$-cryptoxanthin is catalyzed by HYD, and $\beta$-cryptoxanthin is converted to zeaxanthin by the continued action of HYD. At the late yellowing stage, broccoli heads had higher proportions of $\beta$-cryptoxanthin than the previous samples. Briefly, the effect of $H Y D$ on carotenoid biosynthesis in broccoli was clear and played a primary role in the early stages of yellowing. The evolution of the carotenoid component was consistent with the process of postharvest broccoli coloration.

\section{DEGs involved in flavonoid biosynthesis}

Flavonoids, as secondary metabolites of plants, are important components of plant coloration and contribute to plant resistance to external stress. Suzuki et al..$^{29}$ found that flavonoid biosynthesis in Torenia hybrida could be regulated by inhibiting the expression of $C H S$ and $D F R$ 

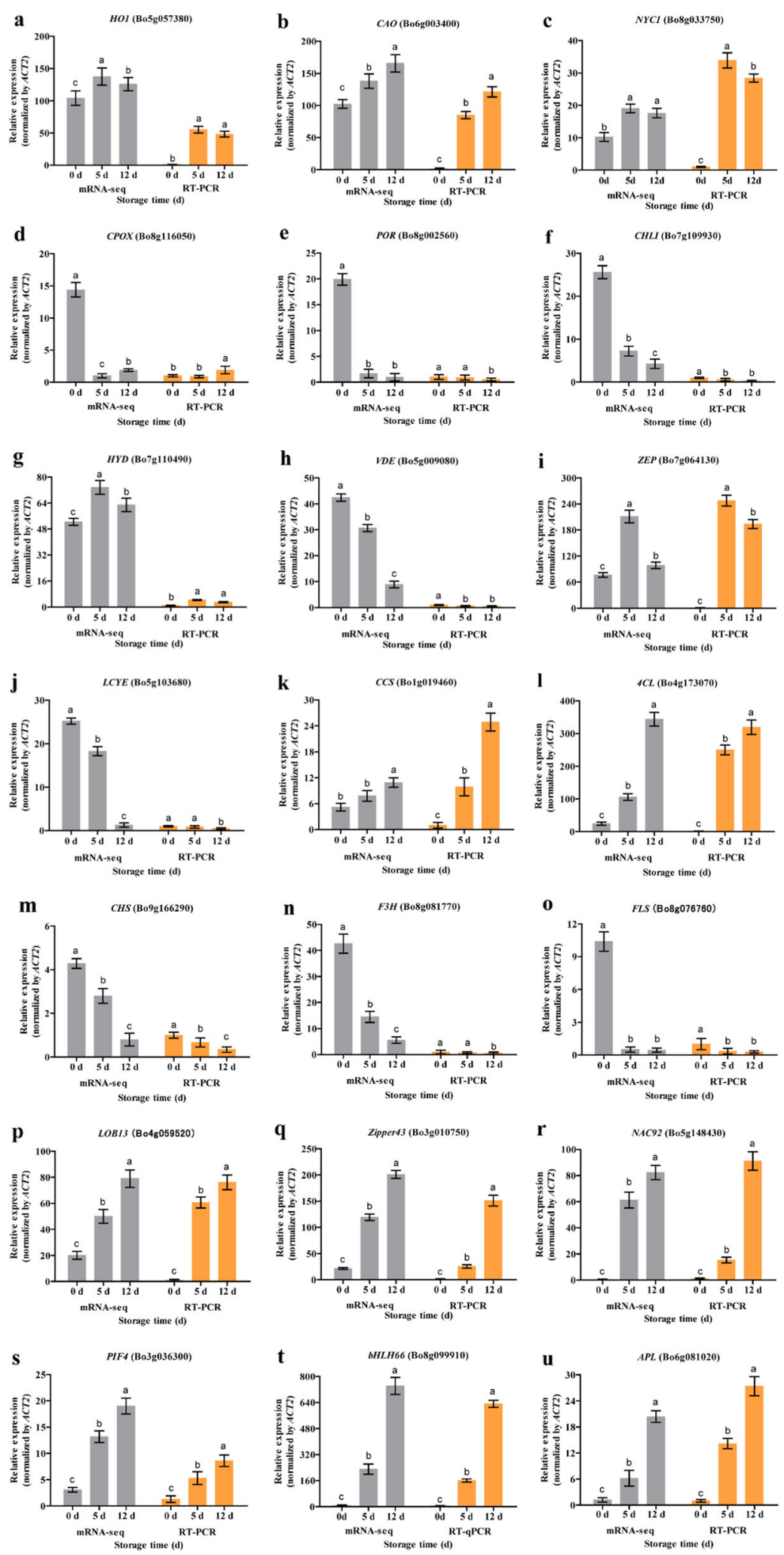

Fig. 3 Comparison of mRNA-seq and RT-PCR analyses for 21 differentially expressed genes (DEGs) during broccoli yellowing. a-u DEGs associated with chlorophyll metabolism $(\mathbf{a}-\mathbf{f})$, carotenoid biosynthesis $(\mathbf{g}-\mathbf{k})$, or flavonoid biosynthesis $(\mathbf{I}-\mathbf{o})$ and TFs $(\mathbf{p}-\mathbf{u})$. The relative expression levels are plotted as FPKM values. The different letters indicate significant differences among the different sampling points $(P<0.05)$, and the error bars indicate the standard deviations 
genes, thereby changing the flower color. Similarly, $\mathrm{Ar}^{30}$ et al. certified that CHS and DFR genes were directly related to the coloration of petunias. In our study, four candidate DEGs associated with flavonoid biosynthesis were identified, most of which were downregulated during the process of broccoli yellowing. These genes included CHS, FLS, and F3H, as well as the DEGs encoding 4CL that included both downregulated and upregulated genes. The decrease in flavonoid content in the early stages of broccoli yellowing may be caused by flavonoid consumption in postharvest broccoli in response to stress. However, the flavonoid content increased slightly in the late yellowing stage of broccoli, probably due to the upregulation of $4 C L$ (Bo4g173070), offsetting the downregulation of $C H S, F L S$, and $F 3 H$ as $4 C L$ downstream genes. The decreased expression of DEGs in flavonoid biosynthetic pathways and diminishing flavonoid content during postharvest storage verified that flavonoids were not directly related to the yellow coloration in broccoli.

\section{TFs involved in broccoli yellowing}

Based on the above research on pigment and related metabolic pathway genes in broccoli, we inferred that the degradation and biosynthesis of pigments occurred throughout the entire process of yellowing in a dynamic form that was directly related to some TFs of key genes. Meng et al. ${ }^{31}$ found that Bell-like homeodomain 11 plays a key role in chloroplast development and chlorophyll synthesis in tomato fruit. MYC2/3/4 and NAC19/55/72 directly activate major chlorophyll catabolic genes in jasmonic acid-mediated Arabidopsis. Furthermore, ethylene promotes EIN3- and ORE1-mediated regulation of chlorophyll catabolic genes in Arabidopsis ${ }^{32}$. From the data for the TF GO categories, three candidate TF families involved in the chlorophyll metabolism pathway were identified: the bZip, bHLH, and MYB families. bZips are involved in the regulation of plant stress resistance ${ }^{33}$ and may induce the regulation of key genes during broccoli yellowing. bHLH transcription factors not only are essential for plant development but also participate in the process of plant coloration. PIF1 is a critical bHLH regulator of chlorophyll biosynthesis ${ }^{34}$. Studies have shown that PIF4, as a bHLH family plant pigment-interacting protein factor, could also be involved in chlorophyll metabolism ${ }^{35}$. MYBs are widely involved in plant development, metabolic regulation, and plant hormone signal transduction $^{36}$, and complex metabolic activities continue to occur in broccoli after harvest.

Previous studies have shown that ERFs, NACs, EINs, and MYBs participate in the carotenoid biosynthetic process and directly regulate carotenoid accumulation. During papaya fruit ripening, EIN3a and $\mathrm{NAC}^{37}$ synergistically regulate carotenoid accumulation and NAC1 activates the $P D S 2 / 4$ gene to modulate carotenoid biosynthesis. ERF6 plays an important role in carotenoid accumulation during tomato fruit ripening ${ }^{38}$. In kiwi fruit, MYB7 regulates chlorophyll and carotenoid accumulation $^{39}$. In our study, the NAC, bHLH, bZip, MYB, and ERF families were the major TF families involved in the regulation of the broccoli carotenoid biosynthetic process. NACs and ERFs are the upstream TFs that regulate the ethylene signaling pathway ${ }^{40}$, which promotes senescence in climacteric plants (e.g., broccoli) by regulating ethylene synthesis, leading to carotenoid accumulation. Moreover, the multihormone pathway is actively initiated by NACs; the ABA signal transduction pathway starts at a branch of the carotenoid biosynthetic network ${ }^{41}$. In the $0 \mathrm{~d}$ vs. $5 \mathrm{~d}$ set, we found that NACs were upregulated, while over $70 \%$ of AP2/ERFs were downregulated. We hypothesized that NACs are the positive regulators of key genes in carotenoid biosynthesis in the early stage of yellowing, whereas AP2/ERFs are actively involved as negative regulators in the accumulation of carotenoids. These explanations suggest that NACs and ERFs are the main TF regulators of carotenoid biosynthesis. Recently, bHLH was found to regulate the biological pathways of bioactive components. Studies have shown that bHLHs and MYBs are involved in flavonoid biosynthesis ${ }^{42}$. We can conclude that carotenoids, which are biologically active components, may also be involved in the regulation of the bHLH and MYB families.

Based on the results from the TF GO categories, we found that bHLH66, PIF4, LOB13, NAC92, and APL were simultaneously enriched in the categories of chlorophyll biosynthesis, regulation of chlorophyll biosynthesis, and carotenoid biosynthetic process. We speculated that $C A O$ and $H Y D$ expression might be regulated or coregulated by bHLH66, PIF4, LOB13, NAC92, and APL. Our results showed that the expression levels of $C A O, H Y D, L O B 13$, bHLH66, PIF4, NAC92, and APL genes were positively correlated. These TFs might have a positive regulatory relationship with the $C A O$ and $H Y D$ genes; this is an area with potential for further research.

Although the flavonoids in this study were not the main factors in broccoli yellowing, the flavonoid biosynthetic pathway contains many important metabolic pathways related to coloration. In our study, the TFs associated with flavonoid biosynthesis were MYBs, NACs, WRKYs, MADSs, and bZips. Numerous reports have demonstrated that the MYB and bHLH TF families participate in flavonoid biosynthesis ${ }^{43}$. Previous studies showed that $\mathrm{CHS}$ in apples ${ }^{44}$ is regulated by MYB4 and MYB5. In strawberries $^{45}$, anthocyanin and flavonoid compound accumulation is regulated by FcMYB1. Li et al. ${ }^{20}$ found that MYB39 and bHLH25 are the major TFs that regulate flavonoid biosynthesis and affected leaf coloration. Moreover, in Arabidopsis thaliana, MYB75, MYB90, MYB113, MYB114, and bHLH played crucial roles in 
regulating the expression of DFR, ANS, and UFGT, affecting flavonoid biosynthesis ${ }^{46}$. WRKYs are involved in the biosynthetic pathway regulating secondary metabolism in plants. A TTG2-like WRKY TF was reportedly involved in the regulation of vacuolar transport and flavonoid biosynthesis ${ }^{47}$. Jaakola et al. ${ }^{48}$ verified that MADS regulates the accumulation of anthocyanin in bilberry fruit. Broadly, our findings provide new insight into the intricate transcriptional regulatory network of broccoli yellowing. With continuous improvement in our understanding of the regulatory network, the perspective of further research needs to be clarified. In our next study, we shift our research to focus on exogenous treatment and plant hormone signaling in the process of broccoli yellowing.

\section{Conclusions}

The objective of this study was to reveal the dynamic process of broccoli yellowing from a transcriptomic perspective. Broccoli stored at $10^{\circ} \mathrm{C}$ began to yellow on postharvest day 5 , with severe yellowing by day 12 . During the yellowing process, the Fv/Fm and NPQ values showed clear responses to the changes in broccoli coloration. Chlorophyll metabolism and carotenoid biosynthesis played vital roles at different stages of yellowing. The transcriptome sequencing and RT-PCR results indicated that $C A O$ and $H Y D$ were the key genes involved in chlorophyll $a$ decline and carotenoid accumulation, respectively, and that these genes were potentially regulated simultaneously by the TFs bHLH66, PIF4, LOB13, NAC92, and APL. The DEGs involved in flavonoid biosynthesis were downregulated, and no direct association between flavonoid content and broccoli coloration was found. The measurements of the chlorophyll, carotenoid, and flavonoid contents during the yellowing process confirmed the reliability of the transcriptome data.

\section{Materials and methods}

\section{Plant material and treatments}

"Naihan-Youxiu" broccoli was harvested by cutting at the base of the stem in Jinzhou, Liaoning, China. The bottom of the picked broccoli retained 3-4 leaves. The picking criteria were based on similar maturity and size and a lack of physical damage and pests. The harvested products were placed in plastic boxes and transported to the laboratory at $20^{\circ} \mathrm{C}$ for $40 \mathrm{~min}$. After precooling until the temperature of the center of the flower balls reached $0{ }^{\circ} \mathrm{C}$, the samples were placed in a polyethylene bag (thickness: $0.03 \mathrm{~mm}$ ) at $80 \%$ relative humidity in the dark. The broccoli was stored at $10^{\circ} \mathrm{C}$, which was determined to be the most suitable temperature for the study of the yellowing mechanism in our previous study, for $15 \mathrm{~d}$. All data for this study were measured at three sampling points: fresh $(0 \mathrm{~d})$, mildly yellowed $(5 \mathrm{~d})$, and severely yellowed (12 d). Samples were taken from the broccoli heads and were immediately frozen in liquid nitrogen and stored at $-80^{\circ} \mathrm{C}$ for analysis. In addition, fresh broccoli was kept for daily sensory color and chlorophyll fluorescence detection. Each experiment was performed three times with three biological replicates each $(n=9)$.

\section{Measurement of chlorophyll fluorescence}

The broccoli heads were placed on the console, and light was blocked with a black curtain. The samples were dark-adapted for $15 \mathrm{~min}$ at $20^{\circ} \mathrm{C}$, and chlorophyll fluorescence was then measured using an imaging fluorometer (Open FluorCam FC 800-O/2020; Photon System Instruments, Brno, Czech Republic). The light sources comprised actinic 1 LED panels (610-620 nm, SL3500$761 / 762$ ), actinic 2 superlight LED panels (4750 K, SL3500-757/758), and an additional PAR/NDVI light panel $(655 / 735 \mathrm{~nm}$, SL3500-763). Images were captured using a CCD-695 camera under the optimum light saturation intensity for broccoli. The images $(820 \times 560$ pixels) and the $\mathrm{Fv} / \mathrm{Fm}$ and NPQ values were processed using FC800-313 software (ver. 0.21.2.0) (Photon System Instruments, Brno, Czech Republic). All images and data for each measurement were automatically repeated six times. Samples from each sampling day with similar Fv/ Fm levels were screened for subsequent transcriptome sequencing.

\section{Measurement of the chlorophyll, carotenoid, and flavonoid contents \\ Chlorophyll extraction and determination}

The methods for the extraction and determination of chlorophyll $a$ and $b$ from broccoli were modified based on those of Mínguez-Mosquera et al. ${ }^{49}$. Five grams of freshly frozen tissue from each of the three samples was ground into powder with liquid nitrogen. Next, the powder samples were homogenized with $\mathrm{MgCO}_{3}$ and $10 \mathrm{~mL}$ of acetone at $4{ }^{\circ} \mathrm{C}$ for $3 \mathrm{~h}$. The solution was washed with acetone five times until the tissues turned yellowish. Acetone $(10 \mathrm{~mL})$ was mixed with the extraction solution, and the homogenized solution was then filtered using filter paper. Subsequently, $10 \mathrm{~mL}$ of $\mathrm{NaCl}$ solution $(10 \%$, $\mathrm{w} / \mathrm{v}$ ) was added to the filtrate, and the aqueous phase was removed using a separatory funnel. The separation liquid was washed with $\mathrm{Na}_{2} \mathrm{SO}_{4}(20 \% \mathrm{w} / \mathrm{v})$, and $10 \mathrm{~mL}$ of diethyl ether was added to isolate the liquid. The concentrate was obtained by rotary evaporation of the organic phase. The concentrated solution was mixed with $2 \mathrm{~mL}$ of acetone and passed through a $0.22 \mu \mathrm{m}$ nylon filter. Finally, the extract was used for HPLC-MS (high-performance liquid chromatography-mass spectrometry) analysis.

HPLC-MS analysis was performed with a liquid chromatograph mass spectrometer (LCMS-8050, Shimadzu, Kyoto, Japan). All LC-MS analyses used the same source 
conditions, polarity, and experimental methods, namely, ESI, positive ion-mode, and MRM-type, respectively. The detailed parameters for each analyte in the MRM experiment are shown in Table S3. The injection volume was $35 \mu \mathrm{L}$, and the flow rate was set to $1.0 \mathrm{~mL} / \mathrm{min}$. A stainless steel $(75 \times 2.1 \mathrm{~mm})$ column, which was packed with $2 \mu \mathrm{m}$ C18 Spherisorb (Shim-pack GIST Series C18; Shimadzu, Kyoto, Japan), was used at $40^{\circ} \mathrm{C}$. The mobile phase solvent system was composed of solutions A and B. The mobile phase was water:ammonium acetate $(0.05 \mathrm{M})$ : methanol (1:1:8 by volume), and solution $B$ was methanol: acetone ( $1: 1$ by volume). The gradient scheme was initially $75 \% \mathrm{~A}$ and $25 \% \mathrm{~B}$ for $5 \mathrm{~min}$, followed by $25 \% \mathrm{~A}$ for $8 \mathrm{~min}$, isocratic for $2 \mathrm{~min}, 10 \%$ A for $8 \mathrm{~min}$, and $100 \%$ B for $5 \mathrm{~min}$, before a return to the initial conditions for $7 \mathrm{~min}$. After column equilibration, chlorophyll $a$ and $b$ in the samples were separated and quantified using standard calibration curves (Table S4). The standards were obtained from Dingguo Changsheng Biotechnology Co. Ltd. (Beijing, China). Three biological replicates were used for each treatment, and the experiments were performed three times.

\section{Carotenoid extraction and HPLC-MS analysis}

The extraction and HPLC-MS analysis of zeaxanthin, $\beta$-carotene, lutein, and $\beta$-cryptoxanthin were performed based on the methods of Luo et al. ${ }^{50}$. The equipment conditions used in the HPLC-MS experiment were the same as those used for the chlorophyll content analysis. The detailed parameters for each analyte in the MRM experiment were optimized as shown in Table S3. The injection volume and flow rate were $10 \mathrm{~mL}$ and $0.2 \mathrm{~mL} /$ min, respectively. Acetonitrile:methanol $(0.1 \mathrm{M}$ ammonium formate):dichloromethane:water $\quad$ (65:20:6:9 by volume) was the mobile phase solvent system, and each run terminated at an isocratic flow rate. The carotenoid contents were calculated using a calibration curve (Table S4). The carotenoid component (zeaxanthin, $\beta$-carotene, $\beta$-cryptoxanthin, and lutein) contents were expressed as $\mathrm{mg}$ of the pure standard equivalent per $\mathrm{kg}$ of fresh weight (FW). The standards were obtained from Dingguo Changsheng Biotechnology Co. Ltd. Three biological replicates were used for each treatment, and the experiments were performed three times.

\section{Determination of flavonoid content}

The flavonoid content was determined as described by Luo et al. ${ }^{50}$. Standard solutions of quercetin at different concentrations were obtained by repeating the same procedure. The quercetin standard was obtained from Dingguo Changsheng Biotechnology Co. Ltd. The flavonoid content was calculated using a calibration curve (Table S4). The total flavonoid content was expressed as $\mathrm{mg}$ of quercetin equivalent/g of fresh weight (FW). Three biological replicates were used for each treatment, and the experiments were performed three times.

\section{RNA extraction, cDNA library preparation, and real-time quantitative RT-PCR}

Buds of broccoli were frozen in liquid nitrogen. An OminiPlant RNA kit (DNase I) (CWBIO, Beijing, China) was used to extract total RNA from broccoli sprouts, and a HiFiScript cDNA Synthesis kit (CWBIO) was used for qRT-PCR to reverse transcribe the extracted total RNA. Following the UltraSYBR Mixture (Low ROX) (CWBIO) manufacturer's instructions, qRT-PCR (QuantStudio 5; Thermo Scientific, Waltham, MA, USA) was used to analyze gene expression in the cDNA template $(20 \mu \mathrm{L})$ reaction. Before sample loading, the cDNA concentration was determined using a spectrophotometer (NanoDrop 2000; Thermo Scientific, Waltham, MA, USA). The gene expression analysis included chlorophyll catabolism genes, carotenoid biosynthesis genes, and flavonoid biosynthesis genes that were associated with color changes. In addition, the gene expression of the relevant transcription factors (the LOB13, Zipper43, NAC92, PIF4, bHLH66, and APL genes) was determined. Primer Premier 5.0 (Premier Biosoft Inc., California, USA) was used to design the primers. The primers used for qRT-PCR are listed in Table S5. Relative gene expression values were normalized to that of the ACT2 gene. The expression of each gene was determined using three biological replicates and calculated using the $2^{-\Delta \Delta C T}$ method.

\section{Illumina deep sequencing and identification of differentially expressed genes (DEGs)}

Three biological replicate RNA samples from fresh broccoli samples and from samples with slight and severe yellowing were prepared for transcriptome sequencing experiments. The RNA concentration was measured using the NanoDrop 2000 (Thermo Scientific). RNA integrity was assessed using an RNA Nano 6000 Assay Kit on the Agilent Bioanalyzer 2100 system (Agilent Technologies, Santa Clara, CA, USA). RNA integrity assessment was followed by library preparation for transcriptome sequencing. cDNA library construction and sequencing were performed by Biomarker Biotechnology (Beijing, China). The libraries were sequenced on an Illumina HiSeq 4000 platform (Illumina, San Diego, CA, USA). To ensure high-quality data, we removed the adapter sequences and low-quality sequence reads from the data sets (including reads containing more than $10 \%$ $\mathrm{N}$ and reads with a quality score of $Q \leq 10$ for more than $50 \%$ of the entire read). The clean reads were mapped to the Brassica oleracea genomic database (ftp://ftp. ensemblgenomes.org/pub/plants/release-25/fasta/brassica_oleracea/) using TopHat2 (ver. 2.0.4) software with the default parameters. Quantification of the gene expression 
levels was estimated as FPKM. Cufflinks (ver. 2.2.1) software was used in the process of quantifying gene expression, and the counts were then normalized to the FPKM values.

DESeq (ver. 1.18.0) was used to identify genes that were differentially expressed during broccoli yellowing. A fold change (FC) value of $\geq 4$ and a false discovery rate (FDR) of $<0.001$ were used as criteria for selecting DEGs. Gene function was annotated based on the GO (http://www. geneontology.org/) and Kyoto Encyclopedia of Genes and Genomes (KEGG, http://www.genome.jp/kegg/) databases. A heat map was generated using $\mathrm{R}$ (ver. 3.5.1) software.

\section{Data analysis}

SPSS v.20 was used for statistical analyses (SPSS Inc., Chicago, IL, USA). Data from the three biological replicates and three parallel experiments were analyzed using standard deviation analysis. Differences between groups were evaluated by significant difference analysis (Duncan's multiple range test). Graphs were prepared using Prism 5 software (GraphPad Inc., La Jolla, CA, USA) and Adobe Illustrator software (CC 2017; Adobe Inc., San Jose, USA).

\section{Acknowledgements}

We thank the agricultural college (Shenyang Agricultural University) for chlorophyll fluorescence determination. This work was supported by the National Key R\&D Program of China (no. 2016YFD0400103).

\section{Authors' contributions}

F.L. and S.-J.J. participated in the experimental design and the improvement of the manuscript. X.-M.K. and Q.Z. contributed to the analysis of the transcriptome data. J.-H.C., X.Z., and Y.-B.Z performed the experimental work. The authors of the study have checked the content of the manuscript to ensure accuracy.

\section{Data availability}

The data generated and analyzed during the current study are available in the NCBI repository.

\section{Conflict of interest}

The authors declare that they have no conflict of interest.

Supplementary Information accompanies this paper at (https://doi.org/ 10.1038/s41438-019-0155-1).

Received: 29 January 2019 Revised: 6 April 2019 Accepted: 12 April 2019 Published online: 01 June 2019

\section{References}

1. Angela, R. et al. Cytosolic and plastoglobule-targeted carotenoid dioxygenases from Crocus sativus are both involved in $\beta$-ionone release. J. Biol. Chem. 36, 24816-24825 (2008).

2. Maldonado-Torres, R. et al. Morphological changes in leaves of Mexican lime affected by iron chlorosis. J. Plant Nutr. 4, 615-628 (2006).

3. Cadrain, D. Leaf yellowing and anthocyanin accumulation are two genetically independent strategies in response to nitrogen limitation in Arabidopsis thaliana. Plant Cell Physiol. 1, 74-93 (2006).

4. Thomas, H. Catabolism of chlorophyll in vivo: significance of polar chlorophyll catabolites in a non-yellowing senescence mutant of Festuca pratensis Huds. New Phytol. 1, 3-8 (2010).
5. Popovsky, S. \& Paran, I. Molecular genetics of the y locus in pepper: its relation to capsanthin-capsorubin synthase and to fruit color. Theor. Appl. Genet. 101, 86-89 (2000).

6. Rodrigo, M. J. Characterization of pinalate, a novel Citrus sinensis mutant with a fruit-specific alteration that results in yellow pigmentation and decreased ABA content. J. Exp. Bot. 54, 727-738 (2003).

7. Gong, Y. \& Mattheis, J. P. Effect of ethylene and 1-methylcyclopropene on chlorophyll catabolism of broccoli florets. Plant Growth Regul. 40, 33-38 (2003).

8. Wang, H. W. et al. Influence of modified atmosphere on induction and activity of respiratory enzymes in broccoli florets during the early stage of postharvest storage. J. Agr. Food Chem. 7, 302-318 (2017).

9. Nomura, M. et al. Effect of reactive oxygen species on quality maintenance of broccoli florets with electrostatic atomized water particle treatment. Food Chem. 237, 749-755 (2017).

10. Dedolph, R. R. et al. Effect of N6-benzylaminopurine on respiration and storage behavior of broccoli (Brassica oleracea var. italica). Plant Physiol. 37, 509-512 (1962).

11. Guo, F. et al. Transcriptomic analysis of differentially expressed genes in an orange-pericarp mutant and wild type in pummelo (Citrus grandis). BMC Plant Biol. 15, 44-72 (2015).

12. Wei, $\mathrm{H}$. et al. Comparative transcriptome analysis of genes involved in anthocyanin biosynthesis in the red and yellow fruits of sweet cherry (Prunus avium L.). PLOS ONE 10, e0121164 (2015).

13. Elsharkawy, I. et al. Transcriptome analysis of an apple (Malus domestica) yellow fruit somatic mutation identifies a gene network module highly associated with anthocyanin and epigenetic regulation. J. Exp. Bot. 66, 7359-7376 (2015).

14. Wang, Y. et al. Physiological and transcriptomic analyses of a yellow-green mutant with high photosynthetic efficiency in wheat (Triticum aestivum I.). Funct. Integr. Genom. 18, 1-20 (2018).

15. Hong, M. et al. Transcriptomic analysis of seed coats in yellow-seeded Brassica napus reveals novel genes that influence proanthocyanidin biosynthesis. Front. Plant Sci. 8, 123-148 (2017).

16. Liu, M. S. et al. Proteomics and transcriptomics of broccoli subjected to exogenously supplied and transgenic senescence-induced cytokinin for amelioration of postharvest yellowing. J. Proteom. 93, 133-144 (2013).

17. Allorent, G. et al. UV-B photoreceptor-mediated protection of the photosynthetic machinery in Chlamydomonas reinhardtii. Proc. Natl Acad. Sci. USA 113, 14864-14869 (2017).

18. Rocchetta, I. \& Hendrik, K. Chromium- and copper-induced inhibition of photosynthesis in Euglena gracilis analysed on the single-cell level by fluorescence kinetic microscopy. New Phytol. 182, 405-420 (2009).

19. Hendrik, S. et al. Short- and long-term operation of the lutein-epoxide cycle in light-harvesting antenna complexes. Plant Physiol. 144, 926-941 (2007).

20. Li, W. et al. Cytological, pigment, and transcriptomic analyses of golden leaf coloration in Ginkgo biloba L. Hortic. Res. 5, 23-65 (2018).

21. Pelletier, M. K. \& Shirley, B. W. Characterization of flavonol synthase and leucoanthocyanidin dioxygenase genes in Arabidopsis. further evidence for differential regulation of "early" and "late" genes. Plant Physiol. 113, 1437-1472 (1997).

22. Chen, L. et al. Transgenic broccoli (Brassica oleracea var. italica) with antisense chlorophyllase (BoCLH1) delays postharvest yellowing. Plant Sci. 174, 25-31 (2008).

23. Stocker, R. et al. Antioxidant activities of bile pigments: biliverdin and bilirubin. Method Enzymol. 186, 301-309 (1990).

24. Zhu, L. et al. Heme oxygenase 1 defects lead to reduced chlorophyll in Brassica napus. Plant Mol. Biol. 93, 579-592 (2017).

25. Mahawar, L. \& Shekhawat, G. S. Heme oxygenase: a functionally diverse enzyme of photosynthetic organisms and its role in phytochrome chromophore biosynthesis, cellular signalling and defence mechanisms. Plant Cell Environ. 8, 125-176 (2017).

26. Antonisamy, P. et al. Anti-inflammatory activity of rhein isolated from the flowers of Cassia fistula L. and possible underlying mechanisms. Saudi J. Biol. Sci. 12, 1319-1341 (2017).

27. Hirschberg, L. Carotenoid biosynthesis in flowering plants. Curr. Opin. Plant Biol. 4, 210-218 (2001)

28. Lois, L. M. et al. Carotenoid biosynthesis during tomato fruit development: regulatory role of 1-deoxy-D-xylulose 5-phosphate synthase. Plant J. 22, 503-513 (2000).

29. Suzuki, K. I. et al. Flower color modifications of Torenia hybrida by cosuppression of anthocyanin biosynthesis genes. Mol. Breed. 6, 239-246 (2000). 
30. Ar, V. D. K. et al. Flavonoid genes in petunia: addition of a limited number of gene copies may lead to a suppression of gene expression. Plant Cell $\mathbf{2}$ 291-299 (1990).

31. Meng, L. et al. BEL1-LIKE HOMEODOMAIN 11 regulates chloroplast development and chlorophyll synthesis in tomato fruit. Plant J. Cell Mol. Biol. 94, 23-51 (2018).

32. Qiu, K. et al. EIN3 and ORE1 accelerate degreening during ethylene-mediated leaf senescence by directly activating chlorophyll catabolic genes in Arabidopsis. PLoS Genet. 11, e1005399 (2015).

33. Amir, M. H. et al. The bZIP transcription factor OsABF1 is an ABA responsive element binding factor that enhances abiotic stress signaling in rice. Plant Mol. Biol. 72, 557-565 (2010).

34. Huq, E. et al. Phytochrome-interacting factor 1 is a critical bHLH regulator of chlorophyll biosynthesis. Science 305, 1937-1941 (2004).

35. Kumar, S. V. et al. Transcription factor PIF4 controls the thermosensory activation of flowering. Nature 484, 242-256 (2012).

36. Dubos, C. MYB transcription factors in Arabidopsis. Trends Plant Sci. 15, 573-581 (2010).

37. Fu, C. C. et al. Papaya CpEIN3a and CpNAC2 co-operatively regulate carotenoid biosynthesis-related genes CpPDS2/4, CpLCY-e and CpCHY-b during fruit ripening. Plant Cell Physiol. 4, 36-65 (2017).

38. Lee, J. M. et al. Combined transcriptome, genetic diversity and metabolite profiling in tomato fruit reveals that the ethylene response factor SIERF6 plays an important role in ripening and carotenoid accumulation. Plant J. 70 191-204 (2012).

39. Ampomah-Dwamena, C. et al. A kiwifruit (Actinidia deliciosa) R2R3-MYB transcription factor odulates chlorophyll and carotenoid accumulation. New Phytol. 42, 121-162 (2018).

40. Chung, M. Y. et al. A tomato (Solanum lycopersicum) APETALA2/ERF gene, SIAP2a, is a negative regulator of fruit ripening. Plant J. 64, 936-947 (2010).
41. Jensen, M. K. et al. Transcriptional regulation by an NAC (NAM-ATAF1,2-CUC2) transcription factor attenuates ABA signalling for efficient basal defence towards Blumeria graminis f. sp. hordei in Arabidopsis. Plant J. 56, 867-880 (2008).

42. Baudry, A. et al. TT8 controls its own expression in a feedback regulation involving TTG1 and homologous MYB and bHLH factors, allowing a strong and cell-specific accumulation of flavonoids in Arabidopsis thaliana. Plant J. 46, 768-779 (2006).

43. $\mathrm{Xu}, \mathrm{W}$. et al. Transcriptional control of flavonoid biosynthesis by MYB-bHLHWDR complexes. Trends Plant Sci. 20, 176-185 (2015).

44. Verwoerd, W. S. \& Clark, S. T. A systems approach to identifying correlated gene targets for the loss of color pigmentation in plants. BMC Bioinforma. 12 343-343 (2011)

45. Salvatierra, A. et al. Increased accumulation of anthocyanins in Fragaria chiloensis, fruits by transient suppression of FCMYB1 gene. Phytochemistry $\mathbf{9 0}$, 25-36 (2013).

46. Gonzalez, A. et al. Regulation of the anthocyanin biosynthetic pathway by the TTG1/bHLH/MYB transcriptional complex in Arabidopsis seedlings. Plant J. 53, 814-827 (2010)

47. Amato, A. et al. A grapevine TTG2-like WRKY transcription factor is involved in regulating vacuolar transport and flavonoid biosynthesis. Front Plant Sci. 7, 2-31 (2016).

48. Jaakola, L. et al. A SQUAMOSA MADS-box gene regulates anthocyanin accumulation in bilberry fruits. Plant Physiol. 153, 1619-1629 (2010).

49. Mínguez-Mosquera, M. I. et al. Determination of chlorophylls and carotenoides by high-performance liquid chromatography during olive lactic fermentation. J. Chromatogr. 585, 259-266 (1991).

50. Feng, Luo. et al. Effects of methyl jasmonate and melatonin treatments on the sensory quality and bioactive compounds of harvested broccoli. RSC Adv. 8 , 41422-41431 (2018) 